\title{
Core-Shell Nanostructures of Gold - Copolymer of 3-Carboxy-N-(2- Thenylidene) Aniline and Anthranilic Acid
}

\author{
Rana Golshaei', Sayed Mehdi Ghoreishi' and A Sezai Sarac ${ }^{3 *}$ \\ ${ }^{1}$ University of Kashan, Institute of Nano Science and Nano Technology, Iran \\ 2Department of Analytical Chemistry, Faculty of Chemistry, University of Kashan, Iran \\ ${ }^{3}$ Istanbul Technical University, Department of Chemistry and Polymer Science and Technology, Nanoscience \& \\ Nanoengineering, Istanbul, Turkey
}

\begin{abstract}
In this study, gold/copolymer core-shell nanocomposites as a new metal-polymer composite have been obtained by in situ polymerization. During the polymerization, gold nanoparticles were highly stabilized throughout the copolymer matrix. Fabricated nanocomposites have been characterized by UV-Visible, and Attenuated total reflection - Fourier transform infrared (ATR-FTIR) spectrophotometry. Structure and morphology of synthesized nanoparticles were analyzed by atomic force microscopy (AFM) and Electron Microscopy/Energy-Dispersive X-ray Spectroscopy SEM/EDX. The electro-activity of synthesized Au/copolymer nanoparticles were studied with electrochemical impedance spectroscopy (EIS).
\end{abstract}

\section{Keywords}

In Situ Polymerization, Gold nanoparticles, Core/Shell nanoparticles, Electrochemical Impedance Spectroscopy (EIS)

\section{Introduction}

Nowadays, synthesis of metal/polymer nanocomposites as core-shell nanomaterials has potential for accessing a range of new composites with interesting catalytic, conductive, magnetic or optical, which arises from quantum size effects of nanoparticles and processability of matrix polymers [1]. Futhurmore, by immobilizing the metal nanoparticles in polymer matrix, the nanoparticles are protected against aggregation.Gold nanoparticles with unique optical, electrical and chemical properties are versatile materials for a broad range of applications. But in order to overcome the nanoparticles instability problems, modification of nanoparticles with polymers is necessary $[2,3]$. Polyaniline is one of the most popular conductive polymers due to its environmental stability, high conductivity, lowcost of its synthesis and controllable electrical conductivity [4]. But, the low processibility due to its low solubility is a problem [5]. To get rid of this problem, poly aniline derivatives were used, one of them is Poly(anthranilic acid) (PANA), since PANA is more processable due to its solubility in aqueous, non-aqueous solvents and other polar solvents [6], another derivative 3-carboxy- $\mathrm{N}$-(2-thenylidene) aniline was used as a comonomer in this study for the first time. Use of gold (III) chloride as an oxidant for polymer synthesis provides a new route for chemical synthesis of core/shell nanocomposites. Among different methods to synthesize core/shell nanostructures, in situ polymerization as a feasible and fast simple method is prefered which, oxidation of monomer and reduction of metal ions take place simultaneously for forming composite structures (metalpolymer) [7]. Fabricated $\mathrm{Au} /$ copolymer core-shell nanostructures and synthesized nanoparticles were characterized spectroscopically (Fourier Transform Infrared - Attenuated Total Reflectance (FTIRATR) and UV-Vis spectrophotometer) and morphologically (SEM) (Scanning Electron Microscopy)/EDX(Energy-Dispersive X-ray Spectroscopy .Electroactivity of synthesized Au/copolymer was investigated by EIS.

\section{Experimental}

\section{Materials}

3-aminobenzoic acid $\left(\mathrm{C}_{7} \mathrm{H}_{7} \mathrm{NO}_{2}\right)$, Hydrogen aurichloride $\left(\mathrm{HAuCl}_{4}\right)$ and 3-carboxy-N-(2-thenylidene) aniline were obtained from Sigma Aldrich. All the chemicals used were as analytical grade. Double-distilled deionized water was used in all of the experiments. Sodium dodecyl sulfate (SDS) and dimethyl formamide (DMFAnalytical Grade) was purchased from Sigma Aldrich.

\section{Instruments}

The effect of the gold nanoparticles on the resulting $\mathrm{Au} /$ copolymer nanocomposites were characterized by UV-Visible

*Corresponding author: A. Sezai Sarac, Department of Chemistry, Istanbul Technical University, Maslak, 34469 Istanbul, Turkey, E-mail: sarac@itu.edu.tr

Received: July 31, 2015: Accepted: August 28, 2015: Published: September 02, 2015

Copyright: @ 2015 Golshaei R. This is an open-access article distributed under the terms of the Creative Commons Attribution License, which permits unrestricted use, distribution, and reproduction in any medium, provided the original author and source are credited. 


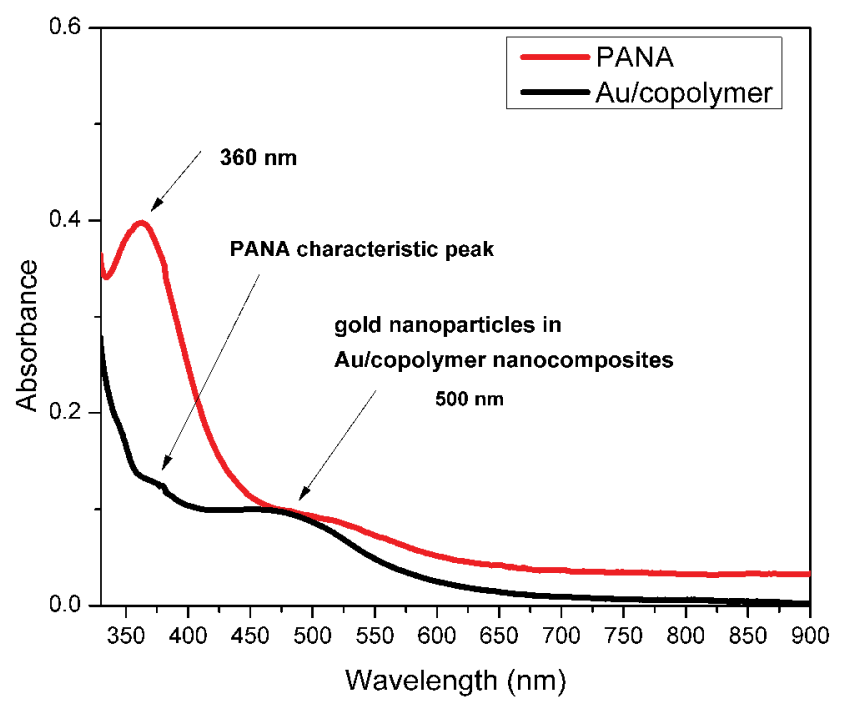

Figure 1: The UV-vis spectra of the PANA and Au/copolymer nanocomposites

(Perkin Elmer, Lambda 45) spectrophotometric analysis.The structural properties of the synthesized Au/copolymer core/shell structures were also characterized by Fourier transform infraredattenuated total reflectance (FTIR-ATR) spectrophotometer (Perkin Elmer, Spectrum One, with a universal ATR attachment with a diamond and ZnSe crystal, USA). Structure and morphology of nanocomposites, were investigated by Atomic Force Microscopy (Nanosurf EasyScan2) in non-contact mode using $\mathrm{Al}$ coated high resonance frequency silicon tips Nanosensors NCRL tips $(40 \mu \mathrm{m}$ width, $225 \mu \mathrm{m}$ length) and SEM (Scanning Electron Microscopy)/ EDX (Energy- Dispersive X-ray Spectroscopy) (QUANTA 400 F). Electrochemical impedance spectroscopic measurements of $\mathrm{Au} /$ copolymer nanocomposites were performed using potentiostat 2263 Electrochemical Analyser (Princeton Applied Research, USA) with frequency range between $0.01 \mathrm{~Hz}$ and $100 \mathrm{kHz}$ and $\mathrm{AC}$ voltage of 10 $\mathrm{mV}$. Three-electrode system, consisting of platinum wire as both a working and counter electrode and silver wire as pseudo reference electrode was used. All measurements were repeated three times for confirmation. Equivalent circuit for the simulation of the EIS spectra of Au/copolymer core/shell nanocomposite solutions was performed with ZSimp Win programme (v.3.10).

\section{Synthesis of Au/copolymer nanoparticles}

$\mathrm{Au} /$ copolymer nanoparticles were synthesized by in-situ polymerization. In the procedure, $200 \mathrm{mg}$ Sodium dodecyl sulfate (SDS) was added to $100 \mathrm{ml}$ of doubly distilled water inside the three-neck round-bottomed flask equipped with stirrer in order to form micelles. After stirring for five hours, 3-aminobenzoic acid $(0.57 \mathrm{~g})$ was added to the flask and stirred in an ultrasonic bath for $30 \mathrm{~min}$, after completely dissolution of this monomer, $0.057 \mathrm{~g}$ of 3-carboxy-N-(2-thenylidene) aniline was added to the solution and stirred under the same conditions in ultrasonic bath. After the dissolution of the monomers, $\mathrm{HAuCl}_{4}(2 \mathrm{ml})$ was added dropwise to the solution and polymerization was initiated and carried out at $28^{\circ} \mathrm{C}$ for about $24 \mathrm{~h}$. Then the precipitated brown polymer has been collected by filtration, washed with methanol and water and dried in vacuum at $80^{\circ} \mathrm{C}$ for two days.

\section{Results and Discussions}

\section{UV Spectrophotometric Analysis}

Incorporation of gold nanoparticles into the polymer matrix was followed by UV-Visible spectrophotometrically after dissolution of $\mathrm{Au} /$ copolymer in DMF (Figure 1). The spectra of pure PANA showed an absorption band in $360 \mathrm{~nm}$ which is characteristic for PANA and corresponds to $\pi-\pi^{\star}$ transition in benzene ring. The spectra of $\mathrm{Au} /$

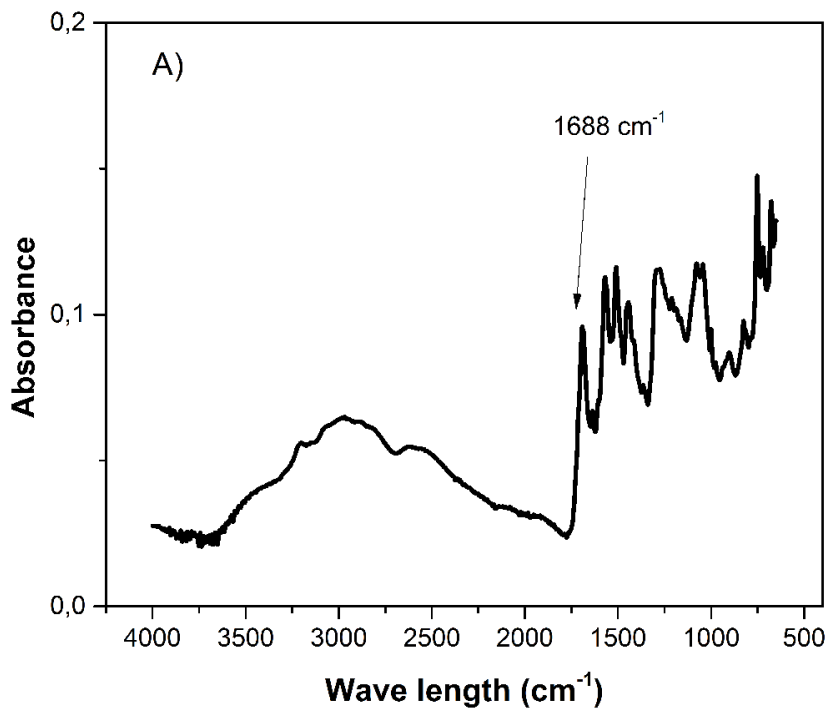

Figure 2A: FTIR-ATR spectra of PANA nanoparticles

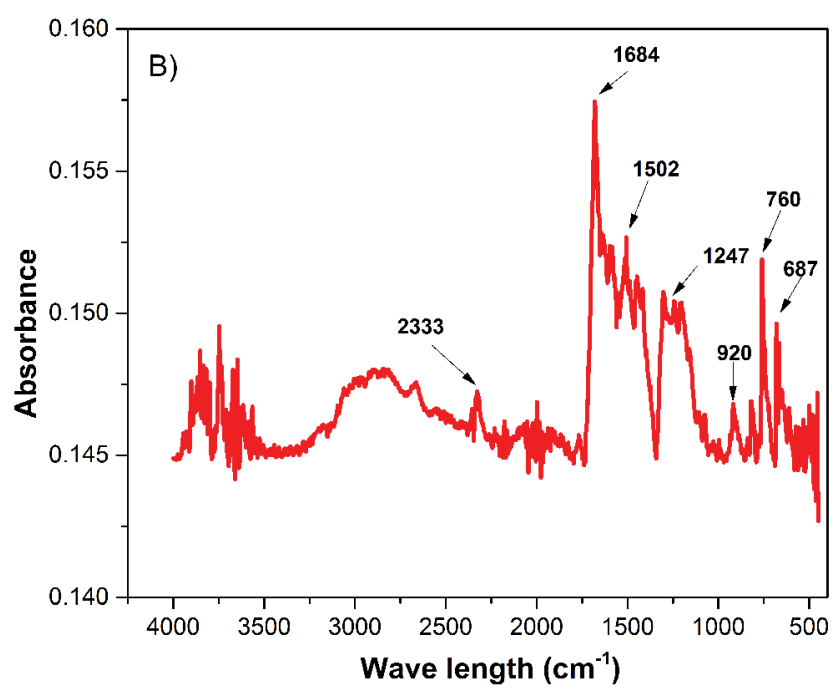

Figure 2B: FTIR-ATR spectra of Au/copolymer nanocomposites

copolymer consists of band with the maxima located at about 500 nm compare to PANA which does not exhibit any obvious peaks in this region. This peak is characteristic for gold nanoparticles since gold nanoparticles within the size range $5-50 \mathrm{~nm}$ show an absorption band in the 520-530 nm region [8] and confirms the incorporation of $\mathrm{Au}$ into the copolymer matrix. In the $\mathrm{Au} /$ copolymer, due to the combination of the PANA and gold nanoparticles, PANA characteristic peak is shifted to higher wavelengths and lower absorbance values.

\section{FTIR-ATR characterization}

The, PANA and $\mathrm{Au} /$ copolymer nanocomposites are characterized by FTIR-ATR spectrophotometer and spectrum is given in Figure 2. The strong stretching vibration of carboxyl group $(\mathrm{C}=\mathrm{O})$ of PANA was at around $1688 \mathrm{~cm}^{-1}$ (Figure 2-A), however it was shifted to 1684 $\mathrm{cm}^{-1}$ in case of $\mathrm{Au} /$ copolymer nanocomposite (Figure 2-B) [9]. In the presence of 3-carboxy-N-(2-thenylidene)aniline, new vibrational bands at around $1247 \mathrm{~cm}^{-1}$ and $1502 \mathrm{~cm}^{-1}$ coresponding to $\mathrm{C}-\mathrm{C}$ or $\mathrm{C}=$ $\mathrm{C}$ stretching of the thiophene ring were appeared. Additionally, S-H stretching of thiophene ring at $2333 \mathrm{~cm}^{-1}$ and strong bands at 687 $\mathrm{cm}^{-1}, 760 \mathrm{~cm}^{-1}$ and $920 \mathrm{~cm}^{-1}$, assigned to the C-S stretching vibration of the thiophene ring, provide incorporation of 3-carboxy-N-(2thenylidene) aniline into the final copolymer composites [10]. 

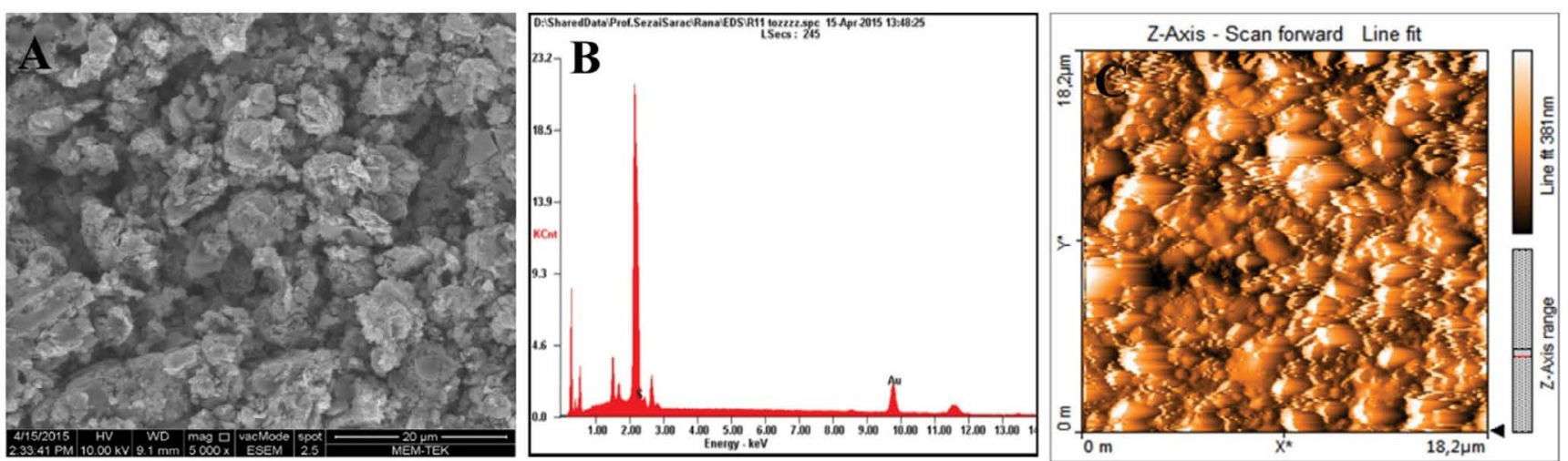

Figure 3: A) SEM B) EDX C) AFM images of Au/copolymer nanocomposites
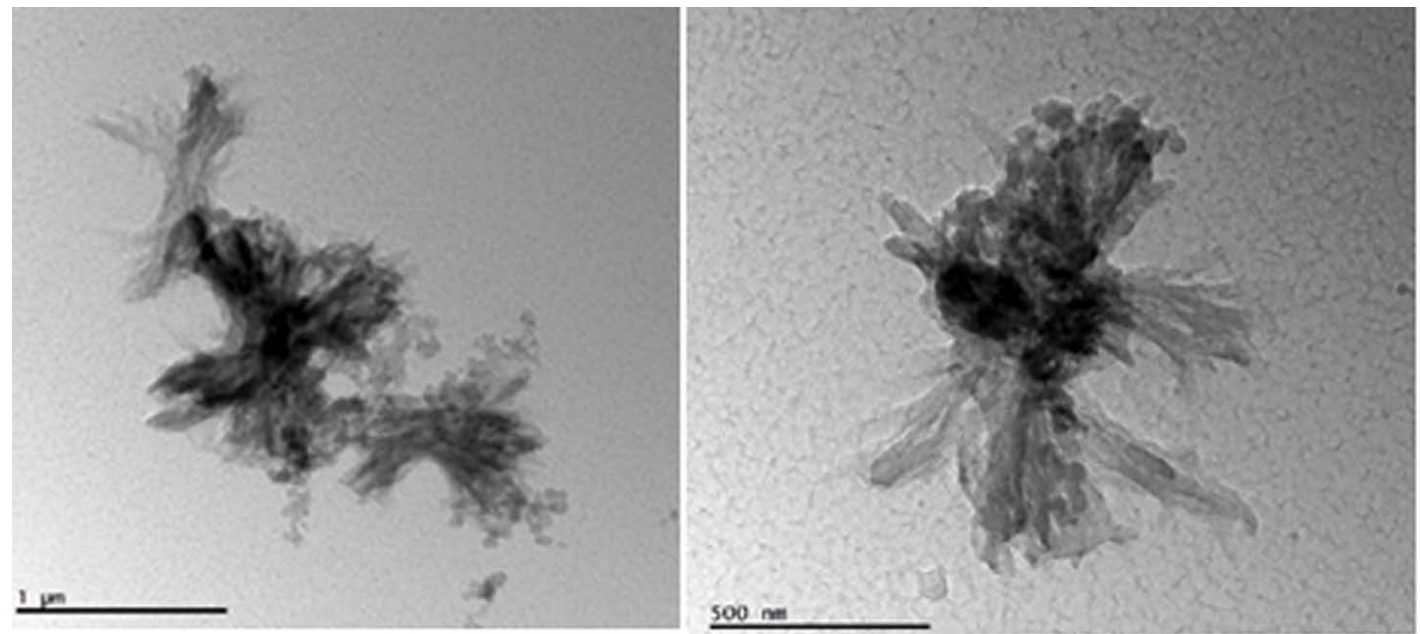

Figure 4: TEM images of Au/copolymer nanocomposites (left 1 micron and right $500 \mathrm{~nm}$ scale)

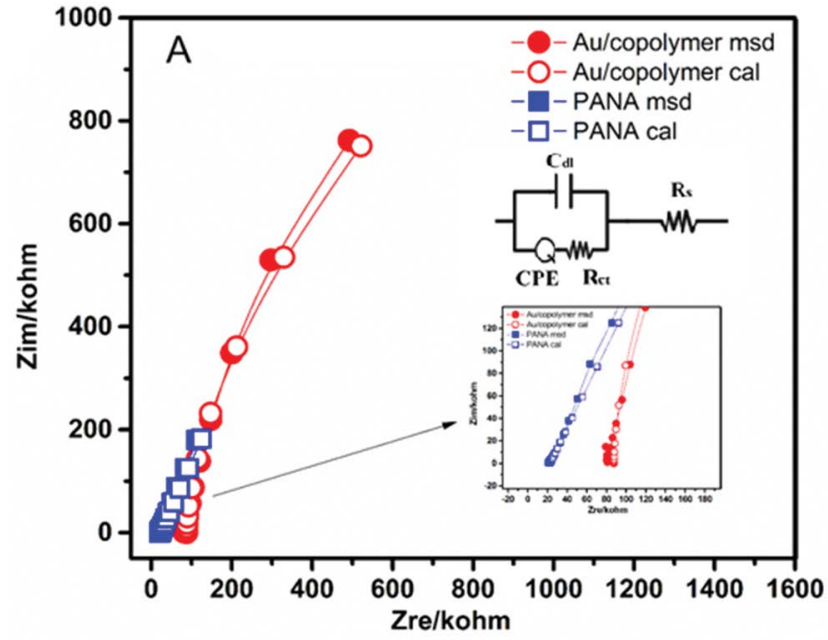

Figure 5A: Calculated and Measured values in A) Nyquist plots of Au/ copolymer and PANA core/shell nanocomposites (Inset Equivalent Circuit)

\section{Morphology and structure of Au/copolymer (SEM, AFM, EDX)}

The morphology of synthesized $\mathrm{Au} /$ copolymer nanocomposites were studied via (Scanning electron microscopy) SEM and (atomic force microscopy) AFM and EDX analyses (Figure 3). SEM /EDX images of $\mathrm{Au} / \mathrm{copolymer}$ nanocomposites revealed the presence of superficial Au nanoparticles homogenously dispersed in copolymer matrix. AFM image of a thin film polymer figure 3-C shows uniform polymeric globules nanocomposite in the copolymer matrix, with average surface roughness of $13 \mathrm{~nm}$ indicates the homogeneity of the film. TEM results also confirmed the core-shell structural formation (Figure 4).

\section{Electrochemical impedance spectroscopy (EIS) and Equivalent Circuit Modelling}

Electrical properties of the solutions of $\mathrm{Au} /$ copolymer nanocomposite were determined by electrochemical impedance spectroscopy (EIS) since it is a sensitive method to investigate the changes on the electrode surface [11]. The Nyquist (Figure 5-A) and Bode phase (Figure 5-B) plots of the Au/copolymer core/shell nanocomposite solutions were given in the frequency range $0.01 \mathrm{~Hz}$ to $100 \mathrm{kHz}$.

The measured impedance spectra were analyzed in terms of electrical equivalent circuits to evaluate the physical model of the system using the analysis program ZSimpWin. The circuits for Au/ copolymer core/shell nanocomposites which describe the physical properties of the system and provide a good fit to the measured data with a reasonable number of circuit elements, were chosen. The calculated and measured data were fitted well together with the chosen equivalent circuits. The impedance spectra for Au/copolymer core/ shell nanocomposites was described best by the equivalent circuit of $\mathrm{R}(\mathrm{C}(\mathrm{RQ})$ ) (Figure 5) in short hand. Table 1 represents the fitting parameters for the equivalent circuit elements by modelling of the impedance spectra. The first component of the circuit, is the solution 
Table 1: Fitting values for the equivalent circuit elements by the simulation of the impedance spectra of PANA and Au/copolymer core/shell nanocomposites.

\begin{tabular}{|l|l|l|l|r|r|}
\hline Samples & $\mathrm{Rs}^{*}(\mathrm{k} \Omega)$ & $\mathrm{C}_{\mathrm{dl}}(\mu \mathrm{F})$ & $\mathrm{Rct}(\mathrm{k} \Omega)$ & $\mathrm{Q}(\mathrm{CPE})\left(\mu \Omega^{-1}\right)$ & $\mathrm{n}$ \\
\hline PANA & 14 & 5.22 & 21.8 & 10.0 & 0.67 \\
\hline Au/copolymer & 60 & 5.80 & 83.0 & 2.7 & 0.85 \\
\hline
\end{tabular}

${ }^{*}$ Rs values were calculated from Nyquist figures.

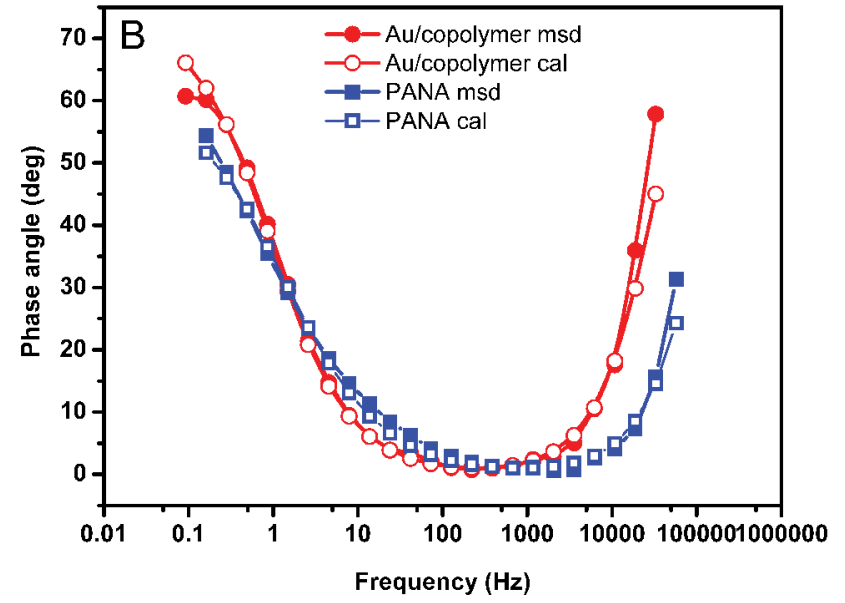

Figure 5B: bode phase plots of PANA and Au/copolymer core/shell nanocomposites

resistance $\left(\mathrm{R}_{\mathrm{s}}\right)$ which corresponds to the ohmic resistance due to the presence of the electrolyte in solution and of electrical contacts [12]. In our case, electrolyte in DMF solution corresponds to $\mathrm{Au} /$ copolymer core/shell nanocomposites. Therefore, solution resistance $\left(\mathrm{R}_{\mathrm{s}}\right)$ is attributed to the resistance between core/shell nanocomposites and size of the Au/copolymer nanoparticles, furthermore PANA carried more charges due to more anionic carboxyl groups existing therein. After formation of Au/copolymer and neutralization of the PANA with $\mathrm{Au}$ ions, the $\mathrm{R}_{\mathrm{s}}$ values can be increased (Table 1).

The second element of the circuit is double layer capacitance $\left(\mathrm{C}_{\mathrm{dl}}\right)$ along $\mathrm{Pt}$ working electrode. It arises from alignment of partially charged $\mathrm{Au} /$ copolymer nanocomposites along electrode surface. The difference between capacitance values of PANA and $\mathrm{Au} /$ copolymer can be explained by the difference of gold and copolymer in terms of structure and size [13]. The second $\mathrm{R}$ in the circuit corresponds to charge transfer resistance $\left(\mathrm{R}_{\mathrm{ct}}\right)$ between $\mathrm{Au} /$ copolymer nanocomposites and $\mathrm{Pt}$ electrode. Presence of gold nanoparticles in the copolymer matrix leads to significant increases of the charge transfer resistance (Rct), compared to the pure PANA. This increase was attributed to the formation of adsorption layer on the Pt electrode-nanocomposite interphase [14], however the whole increase depends on the size, concentration and surface area of formed gold particles after the polymerization of copolymer with $\mathrm{HAuCl}_{4}$ [15]. CPE was applied in the equivalent circuit for the simulation of the impedance data since it takes into account the non-homogeneity of the conductance [12]. The formula $\left(\mathrm{ZC}_{\mathrm{PE}}=\mathrm{T}_{\mathrm{CPE}}(\mathrm{jw})^{-\mathrm{n}}\right)$ is used to define the impedance of a non-ideal electrode where TCPE and $n$ are frequency-independent constants; $\mathrm{w}$ is the angular frequency [11], $\mathrm{n}$ is a parameter describing the deviation from an ideal capacitor and arises from the slope of the $\log \mathrm{Z}$ versus $\log \mathrm{f}$ plot. The values for $\mathrm{n}$ vary from 0 to $1 . \mathrm{n}=1$ subscribes to an ideal capacitor, $(0<\mathrm{n}<$ 1) while $n=0$ and 0.5 , denotes a resistance and Warburg behaviour, respectively [16]. W represents the Warburg impedance and it is attributed to the diffusion of counter-ions. The $n$ values of PANA and $\mathrm{Au}$ /copolymer nanocomposites were 0.67 and 0.84 , respectively indicating that $\mathrm{Au} / \mathrm{copolymer}$ nanostructures behave like capacitors.

\section{Conclusion}

In this study, in situ polymerization of $\mathrm{Au} /$ copolymer core/shell nano-composites were performed. In this method polymerization reaction and the interaction between $\mathrm{HAuCl}_{4}$ and monomers (3-carboxy-N-(2-thenylidene) aniline and Anthranilic acid were done simultaneously. The structural properties of the Au/copolymer core/shells were determined by FTIR-ATR spectrophotometrically. FTIR-ATR and UV-Vis spectrophotometric results evidenced the incorporation of Au nanoparticles into copolymer matrix. At UVVisible spectroscopy, the presence of the characteristic peak of $\mathrm{Au}$ nanoparticles in the wavelength $\sim 500 \mathrm{~nm}$, confirmed the formation of gold nanoparticles in the composite. SEM and EDX images of $\mathrm{Au} /$ copolymer nanocomposites confirmed the dispersion of $\mathrm{Au}$ nanoparticles homogeneously in copolymer matrix. EIS and equivalent circuit modelling of Au/copolymer core/shell nanocomposites indicate that $\mathrm{Au} /$ copolymer nanostructures behave like capacitors.

\section{Acknowledgements}

The authors express their thanks to Scientific and Technological Research Council of Turkey (TUBITAK) for the financial support on project 2216

\section{References}

1. Burda C, Chen X, Narayanan R, El-Sayed MA (2005) Chemistry and properties of nanocrystals of different shapes. Chem Rev 105: 1025-1102.

2. Sahoo Y, Pizem H, Fried T, Golodnitsky D, Burstein L, et al. (2001) Alkyl phosphonate/phosphate coating on magnetite nanoparticles: A comparison with fatty acids, Langmuir, 17: 7907-7911.

3. Lee H, Lee E, Kim do K, Jang NK, Jeong YY, et al. (2006) Antibiofouling polymer-coated superparamagnetic iron oxide nanoparticles as potential magnetic resonance contrast agents for in vivo cancer imaging. J Am Chem Soc 128: 7383-7389.

4. Li D, Huang J, Kaner RB (2009) Polyaniline nanofibers: a unique polymer nanostructure for versatile applications. Acc Chem Res 42: 135-145.

5. Maheshwari V, Saraf RF (2006) High-resolution thin-film device to sense texture by touch. Science 312: 1501-1504.

6. Ming-Sieng Wu, Ten-Chin Wen, A Gopalan (2002) In situ UV-visible spectroelectrochemical studies on the copolymerization of diphenylamine with anthranilic acid. Materials Chemistry and Physics 74: 58-65.

7. Power AC, Betts AJ, Cassidy JF (2011) Non aggregated colloidal silver nanoparticles for surface enhanced resonance Raman spectroscopy. Analyst 136: 2794-2801.

8. Kamat PV (2002) Photo physical,photochemical and photocatalytic aspects of metal nanoparticles. The Journal of Physical Chemistry B, 106: 7729-7744.

9. R. Golshaei, Z. Guler, C. Ünsal, A. S. Sarac, et al. (2015) In situ spectroscopic and electrochemical impedance study of gold/poly (anthranilic acid) core/ shell nanoparticles. European Polymer Journal 66: 502-512.

10. Ohlan A, Singh K, Chandra A, Dhawan SK (2010) Microwave absorption behavior of core-shell structured poly (,4-ethylenedioxy thiophene)-barium ferrite nanocomposites. ACS Appl Mater Interfaces. 2: 927-933.

11. Gu H, Su Xd, Loh KP (2005) Electrochemical impedance sensing of DNA hybridization on conducting polymer film-modified diamond. J Phys Chem B 109: 13611-13618.

12. Giray D, Balkan T, Dietzel B, Sarac AS, (2013) Electrochemical impedance study on nanofibers of poly (m-anthranilic acid)/polyacrylonitrile blends. European Polymer Journal, 49: 2645-2653

13. Zehani N, Dzyadevych SV, Kherrat R, Jaffrezic-Renault NJ4 (2014) Sensitive impedimetric biosensor for direct detection of diazinon based on lipases. Front Chem 2: 44.

14. Sophia I, Gopu G, Vedhi C (2012) Synthesis and Characterization of Poly Anthranilic Acid Metal Nanocomposites. Open Journal of Synthesis Theory and Applications 1:1-8.

15. Bonanni A, Pumera M, Miyahara Y (2011) Influence of gold nanoparticle size (2-50 nm) upon its electrochemical behavior: an electrochemical impedance spectroscopic and voltammetric study. Physical Chemistry Chemical Physics 13: 4980-4986.

16. Lu X, Dou H, Yuan C, Yang S, Hao L, et al. (2012) Polypyrrole/carbon nanotube nanocomposite enhanced the electrochemical capacitance of flexible graphene film for supercapacitors. Journal of Power Sources 197: 319-324. 